Biometrics 59, 721-726

September 2003

\title{
CONSULTANT'S FORUM
}

\section{Goodness-of-Fit Methods for Additive-Risk Models in Tumorigenicity Experiments}

\author{
Debashis Ghosh \\ Department of Biostatistics, University of Michigan, 1420 Washington Heights, \\ Ann Arbor, Michigan 48109-2029, U.S.A. \\ e-mail: ghoshd@umich.edu
}

\begin{abstract}
Summary. In tumorigenicity experiments, a complication is that the time to event is generally not observed, so that the time to tumor is subject to interval censoring. One of the goals in these studies is to properly model the effect of dose on risk. Thus, it is important to have goodness of fit procedures available for assessing the model fit. While several estimation procedures have been developed for current-status data, relatively little work has been done on model-checking techniques. In this article, we propose numerical and graphical methods for the analysis of current-status data using the additive-risk model, primarily focusing on the situation where the monitoring times are dependent. The finite-sample properties of the proposed methodology are examined through numerical studies. The methods are then illustrated with data from a tumorigenicity experiment.
\end{abstract}

KEY WORDS: Additive hazards; Current-status data; Interval censoring; Residual plot; Survival analysis.

\section{Introduction}

In many scientific studies, the outcome of interest is time to a certain event. Most standard methods in survival analysis assume that these times are subject to right censoring. A more complicated situation occurs when the event times cannot be directly observed, but are known to be below or above a random monitoring time. This kind of data is referred to as current-status or interval-censored data.

One setting where current-status data arise is tumorigenicity experiments. Consider the data from a study reported in Dinse and Lagakos (1983). Oral doses of polybrominated biphenyl mixture (PBB), a flame retardant, were administered to 319 Fischer 344 rats over a six-month period, beginning at about seven or eight weeks after birth. When the animal died, either due to natural death or sacrifice, it was determined whether or not bile duct hyperplasia was present. The relationship of interest was the effect of dose level of PBB on the distribution of bile duct hyperplasia in the rats. The dose levels of PBB were measured in milligrams administered per kilogram of body weight and could take on one of six possible values $(0-5)$, corresponding to the actual dose levels of 0 , $0.1,0.3,1.0,3.0$, and 10.0. In addition, three other covariates were measured for each rat: sex, weight at baseline, and the number of the cage in which the rat was housed during the six-month dose period.

While Dinse and Lagakos (1983) were interested in modeling the effect of dose level on tumor prevalence, we might also wish to model the effect of dose on the interval-censored time to tumor development. The proportional hazards model (Cox, 1972 ) is typically used in most survival analysis applications. However, it might not be as appropriate for this context, for there is no biological mechanism to suggest that the effect of dose is multiplicative on the hazard of time to tumor development. Breslow and Day (1987, Chapter 6) argue that in certain instances, an additive model seems more reasonable in modeling the effect of dose on risk.

Understanding the dose-response relationship in the PBB experiment requires proper specification of the effect of dose on time to tumor development. Thus, it is important to have model-checking methods for assessing the fit of the model. Relatively little work has been done with goodness-of-fit procedures for interval censored data. Younes and Lachin (1997) and Farrington (2000) have proposed residuals for the proportional hazards model with interval-censored data. We seek to develop more formal model-checking methods for assessing functional form in the additive-risk model with current-status data.

The course of this article is as follows. In Section 2, we review the procedure of Lin, Oakes, and Ying (1998) for estimation of the additive hazards with current-status data. We then develop goodness-of-fit methods for checking the functional form of covariates in Section 3. In Section 4, we report the results of some simulation studies. The proposed methods are then applied to data from a tumorigenicity experiment in 
Section 5. Finally, we conclude with some brief remarks in Section 6.

\section{Additive-Hazards Model for Current-Status Data}

2.1 Notation and Model

Let $T$ denote the time to the event of interest, let $C$ be the censoring time, and let $\mathbf{Z}$ represent a $p \times 1$ vector of covariates. We assume throughout the article that the covariates are time invariant. It is assumed that $T$ is independent of $C$ given $\mathbf{Z}$. For $i=1, \ldots, n$, the observed data consists of $\left\{C_{i}, \delta_{i}, \mathbf{Z}_{i}\right\}, n$ independent copies from $\{C, \delta, \mathbf{Z}\}$, where $\delta \equiv$ $I(T \geq C)$ indicates that the event of interest has been censored. Note that the event indicator is defined backwards from the usual case of right-censored data.

The effect of $\mathbf{Z}$ on $T$ is formulated through the additive-risk model (Lin and Ying, 1994):

$$
\lambda(t \mid \mathbf{Z})=\lambda_{0}(t)-\beta_{0}^{T} \mathbf{Z},
$$

where $\lambda(t \mid \mathbf{Z})$ is the hazard for $T$ conditional on covariates, $\lambda_{0}(\cdot)$ is an unspecified baseline hazard function, and $\boldsymbol{\beta}_{0}$ is a $p \times 1$ vector of unknown regression coefficients. When the event of interest is rare, the regression parameters in (2.1) are directly interpretable as risk differences associated with a unit change in the covariates. As explained by Breslow and Day (1980), pp. 53-57), a model such as (2.1) can provide useful summaries of excess risk that can complement those given by a proportional hazards model.

\subsection{Estimation}

Lin et al. (1998) proposed an elegant procedure for estimation of $\boldsymbol{\beta}_{0}$ in model (2.1) based on the partial likelihood, using the counting process $N_{i}(t) \equiv \delta_{i} I\left(C_{i} \leq t\right)$ for the $i$ th individual, $i=$ $1, \ldots, n$. Since $N_{i}(t)$ can potentially take one jump, the hazard function corresponding to $N_{i}(t)$ is a well-defined quantity. Let $d H_{i}(t)$ denote this hazard and let $d N_{i}(t)$ be the increment in $N_{i}(t)$. For $d N_{i}(t)$ to equal one, two things must occur. First, $C_{i}$ must equal $t$. Let the hazard of this event be denoted by $d \Lambda^{C}(t)$. Second, the monitoring must reveal that the subject has not experienced the event before time $t$. Under model (2.1), the second event has probability

$$
\begin{aligned}
\operatorname{Pr}\left\{T_{i} \geq t \mid \mathbf{Z}_{i}\right\} & =\exp \left[-\int_{0}^{t}\left\{\lambda_{0}(u)-\boldsymbol{\beta}_{0}^{T} \mathbf{Z}_{i}\right\} d u\right] \\
& =\exp \left\{-\Lambda_{0}(t)+\boldsymbol{\beta}_{0}^{T} \mathbf{Z}_{i}^{*}(t)\right\},
\end{aligned}
$$

where $\Lambda_{0}(t)=\int_{0}^{t} \lambda_{0}(u) d u$ and $\mathbf{Z}_{i}^{*}(t)=t \mathbf{Z}(i=1, \ldots, n)$. The product of the probabilities for these two events yields

$$
d H_{i}(t)=d H_{0}(t) \exp \left\{\boldsymbol{\beta}_{0}^{T} \mathbf{Z}_{i}^{*}(t)\right\},
$$

where $d H_{0}(t)=\exp \left\{-\Lambda_{0}(t)\right\} d \Lambda^{C}(t)$. The model in (2.3) has a form identical to that of the proportional hazards model (Cox, 1972). Consequently, estimation of $\boldsymbol{\beta}_{0}$ can be done based on the partial likelihood.

The preceding discussion has implicitly assumed that censoring or monitoring times for the $n$ individuals come from the same distribution. In situations where the monitoring times are fixed in advance, this is a reasonable assumption. However, there are situations where the monitoring times them- selves depend on the covariates; we refer to this situation as dependent monitoring. In the work by Lin et al. (1998) an estimation procedure for $\boldsymbol{\beta}_{0}$ in (2.1) was proposed for this scenario. They model the censoring or monitoring times by proportional hazards:

$$
d \Lambda^{C}(t \mid \mathbf{Z})=d \Lambda_{0}^{C}(t) \exp \left(\gamma_{0}^{T} \mathbf{Z}\right)
$$

where $d \Lambda^{C}(t \mid \mathbf{Z})$ represents the hazard function for censoring conditional on covariates, $d \Lambda_{0}^{C}(\cdot)$ is an unspecified baseline hazard function, and $\gamma_{0}$ is a $p \times 1$ vector of unknown regression coefficients. For ease of presentation, we have included the same covariate vector $\mathbf{Z}$ in (2.1) and (2.4). The method works with different covariates in the two models as well; we provide an example of this situation in Section 5. Since we are formulating two models, model assessment and selection of them will be important but for different reasons. We discuss this issue at the end of Section 3.

Using arguments similar to those in the first paragraph of this section, we have that

$$
d H_{i}(t)=d H_{0}^{*}(t) \exp \left\{\boldsymbol{\beta}_{0}^{T} \mathbf{Z}_{i}^{*}(t)+\gamma_{0}^{T} \mathbf{Z}_{i}\right\}
$$

with $d H_{0}^{*}(t)=\exp \left\{-\Lambda_{0}(t)\right\} d \Lambda_{0}^{C}(t)$. Lin et al. (1998) suggested a two-stage approach for estimation. The parameters $\gamma_{0}$ are estimated using the partial likelihood estimating function

$$
\mathbf{U}^{*}(\gamma)=\sum_{i=1}^{n}\left\{\mathbf{Z}_{i}-\frac{\sum_{j=1}^{n} Y_{j}\left(C_{i}\right) \mathbf{Z}_{j} \exp \left(\gamma^{T} \mathbf{Z}_{j}\right)}{\sum_{j=1}^{n} Y_{j}\left(C_{i}\right) \exp \left(\gamma^{T} \mathbf{Z}_{j}\right)}\right\}
$$

where $Y_{i}(t)=I\left(C_{i} \geq t\right)(i=1, \ldots, n)$. The maximum partial likelihood estimator $\tilde{\gamma}$ is the solution from setting (2.6) equal to 0. Let $\tilde{\Lambda}_{0}^{C}(t)$ represent the Aalen-Breslow estimator of $\Lambda_{0}^{C}(t)$ in (2.4). The regression parameters $\boldsymbol{\beta}_{0}$ can then be estimated using the estimating function $\mathbf{U}_{\boldsymbol{\beta}}(\boldsymbol{\beta} ; \tilde{\gamma})$ for estimating $\boldsymbol{\beta}_{0}$, where

$$
\mathbf{U}_{\boldsymbol{\beta}}(\boldsymbol{\beta} ; \gamma)=\sum_{i=1}^{n} \int_{0}^{\tau}\left\{\mathbf{Z}_{i}^{*}(t)-\frac{\mathbf{S}^{(1)}(\boldsymbol{\beta}, \gamma, t)}{\mathbf{S}^{(0)}(\boldsymbol{\beta}, \gamma, t)}\right\} d N_{i}(t)
$$

and

$$
\mathbf{S}^{(k)}(\boldsymbol{\beta}, \gamma, t)=n^{-1} \sum_{j=1}^{n} Y_{j}(t) \mathbf{Z}_{j}^{*}(t)^{\otimes k} \exp \left\{\boldsymbol{\beta}^{T} \mathbf{Z}_{j}^{*}(t)+\gamma^{T} \mathbf{Z}_{j}\right\}
$$

$k=0,1$. Let $\tilde{\boldsymbol{\beta}}$ represent the solution to $\mathbf{U}_{\boldsymbol{\beta}}(\tilde{\boldsymbol{\beta}} ; \tilde{\gamma})=\mathbf{0}$. One can show that $n^{-1 / 2} \mathbf{U}_{\boldsymbol{\beta}}\left(\boldsymbol{\beta}_{0} ; \tilde{\gamma}\right)$ and $n^{1 / 2}\left(\tilde{\boldsymbol{\beta}}-\boldsymbol{\beta}_{0}\right)$ converge in distribution to normal random vectors with mean zero and variance-covariance matrices $\mathbf{V}_{\beta} \equiv \boldsymbol{\Sigma}_{\beta}-\boldsymbol{\Sigma}_{\beta \gamma} \boldsymbol{\Omega}_{\gamma}^{-1} \boldsymbol{\Sigma}_{\beta \gamma}^{T}$ and $\boldsymbol{\Sigma}_{\beta}^{-1}-\boldsymbol{\Sigma}_{\beta}^{-1} \boldsymbol{\Sigma}_{\beta \gamma} \boldsymbol{\Omega}_{\gamma}^{-1} \boldsymbol{\Sigma}_{\beta \gamma}^{T} \boldsymbol{\Sigma}_{\beta}^{-1}$, where

$$
\begin{array}{ll}
\boldsymbol{\Sigma}_{\boldsymbol{\beta}} & =\lim _{n \rightarrow \infty} n^{-1} \frac{\partial \mathbf{U}_{\boldsymbol{\beta}}(\boldsymbol{\beta} ; \gamma)}{\partial \boldsymbol{\beta}} \quad \boldsymbol{\Sigma}_{\boldsymbol{\beta} \gamma}=\lim _{n \rightarrow \infty}-n^{-1} \frac{\partial \mathbf{U}_{\boldsymbol{\beta}}(\boldsymbol{\beta} ; \gamma)}{\partial \gamma} \\
\boldsymbol{\Omega}_{\gamma} & =\lim _{n \rightarrow \infty}-n^{-1} \frac{\partial \mathbf{U}^{*}(\gamma)}{\partial \gamma},
\end{array}
$$

and $\boldsymbol{\beta}$ and $\boldsymbol{\gamma}$ are evaluated at $\boldsymbol{\beta}_{0}$ and $\boldsymbol{\gamma}_{0}$, respectively. Corresponding consistent estimators of $\tilde{\boldsymbol{\Sigma}}_{\boldsymbol{\beta}}, \tilde{\boldsymbol{\Sigma}}_{\boldsymbol{\beta} \gamma}$ and $\tilde{\boldsymbol{\Omega}}_{\gamma}$ in (2.8) can 
be computed by replacing their components with the samplebased estimators and $\boldsymbol{\beta}_{0}$ and $\boldsymbol{\gamma}_{0}$ with $\tilde{\boldsymbol{\beta}}$ and $\tilde{\gamma}$.

\section{Goodness-of-Fit Methods}

We now develop numerical and graphical methods for model checking corresponding to the estimation procedures in Section 2 by extending the simulation-based procedure of Lin, Wei, and Ying (1993). The dependent monitoring scenario is considered here, as it seems reasonable to assume that the monitoring times for animals in carcinogenicity experiments are covariate dependent. We will primarily be interested in checking the functional form for the covariates $\mathbf{Z}$; related extensions are discussed in Section 6. We focus on the case where the monitoring times depend on $\mathbf{Z}$ through the proportional hazards model (2.4).

In the dependent-monitoring situation, $M_{i}\left(t ; \gamma_{0}\right)(i=1$, $\ldots, n)$ are martingales, where

$$
\begin{aligned}
& M_{i}\left(t ; \gamma_{0}\right)=N_{i}(t)-\int_{0}^{t} Y_{i}(u) \exp \left\{\boldsymbol{\beta}_{0}^{T} \mathbf{Z}_{i}^{*}(u)+\gamma_{0}^{T} \mathbf{Z}_{i}\right\} d H_{0}^{*}(u) . \\
& \text { Let } \tilde{H}_{0}^{*}(t ; \boldsymbol{\beta}, \gamma)=\sum_{i=1}^{n} \int_{0}^{t} d N_{i}(u) / \mathbf{S}^{(0)}(\boldsymbol{\beta}, \gamma, u) \text { and } \\
& \qquad \begin{aligned}
\tilde{M}_{i}(t ; \tilde{\gamma})= & N_{i}(t)-\int_{0}^{t} Y_{i}(u) \\
& \times \exp \left\{\tilde{\boldsymbol{\beta}}^{T} \mathbf{Z}_{i}^{*}(u)+\tilde{\gamma}^{T} \mathbf{Z}_{i}\right\} d \tilde{H}_{0}^{*}(u ; \tilde{\boldsymbol{\beta}}, \tilde{\gamma}) .
\end{aligned}
\end{aligned}
$$

It is natural to then use $\tilde{M}_{i}(t ; \tilde{\gamma})$ for estimation of $M_{i}\left(t ; \gamma_{0}\right)$.

Define $\tilde{M}_{i} \equiv \tilde{M}_{i}(\tau ; \tilde{\gamma})$. We can plot $\tilde{M}_{i}$ versus $Z_{j i}$ to graphically assess the functional form for the $j$ th covariate. More formal methods can be constructed based on the cumulative $\operatorname{sum} W_{j}(x ; \tilde{\gamma})$ :

$$
W_{j}(x ; \tilde{\gamma})=n^{-1 / 2} \sum_{i=1}^{n} I\left(Z_{j i} \leq x\right) \tilde{M}_{i} .
$$

Let $\mathbf{S}_{\gamma}^{(1)}(\boldsymbol{\beta}, \boldsymbol{\gamma}, t)=\partial / \partial \boldsymbol{\gamma} \mathbf{S}^{(0)}(\boldsymbol{\beta}, \boldsymbol{\gamma}, t)$ and

$$
\begin{aligned}
\mathbf{K}(\boldsymbol{\beta}, \gamma, x)= & n^{-1} \sum_{i=1}^{n} \int_{0}^{\tau} Y_{i}(u) \exp \left\{\boldsymbol{\beta}^{T} \mathbf{Z}_{i}^{*}(u)+\gamma^{T} \mathbf{Z}_{i}\right\} \\
& \times I\left(Z_{j i} \leq x\right)\left\{\mathbf{Z}_{i}-\frac{\mathbf{S}_{\gamma}^{(1)}(\boldsymbol{\beta}, \gamma, u)}{\mathbf{S}^{(0)}(\boldsymbol{\beta}, \gamma, u)}\right\} d \tilde{H}_{0}^{*}(u ; \boldsymbol{\beta}, \gamma) .
\end{aligned}
$$

Using arguments in the Appendix of Lin et al. (2000), the null distribution of $W_{j}(x ; \tilde{\gamma})$ can be approximated by that of the mean-zero Gaussian process $\tilde{W}_{j}(x ; \tilde{\gamma})$ :

$$
\begin{aligned}
& \tilde{W}_{j}(x ; \tilde{\gamma}) \\
& =n^{-1 / 2} \sum_{i=1}^{n} \int_{0}^{\tau}\left\{I\left(Z_{j i} \leq x\right)-\frac{S(\tilde{\boldsymbol{\beta}}, \tilde{\gamma}, u, x)}{\mathbf{S}^{(0)}(\tilde{\boldsymbol{\beta}}, \tilde{\gamma}, u)}\right\} d N_{i}(u) G_{i} \\
& -\mathbf{B}^{T}(\tilde{\boldsymbol{\beta}}, \tilde{\gamma}, x) \tilde{\boldsymbol{\Sigma}}_{\boldsymbol{\beta}}^{-1} n^{-1 / 2} \sum_{i=1}^{n} \int_{0}^{\tau}\left\{\mathbf{Z}_{i}^{*}(u)-\frac{\mathbf{S}^{(1)}(\tilde{\boldsymbol{\beta}}, \tilde{\gamma}, u)}{\mathbf{S}^{(0)}(\tilde{\boldsymbol{\beta}}, \tilde{\gamma}, u)}\right\} d N_{i}(u) G_{i} \\
& -\mathbf{K}^{T}(\tilde{\boldsymbol{\beta}}, \tilde{\gamma}, x) \tilde{\boldsymbol{\Omega}}_{\gamma}^{-1} n^{-1 / 2} \sum_{i=1}^{n} \int_{0}^{\tau}\left\{\mathbf{Z}_{i}-\frac{\mathbf{R}^{(1)}(\tilde{\gamma}, u)}{\mathbf{R}^{(0)}(\tilde{\gamma}, u)}\right\} d N_{i}^{C}(u) G_{i},
\end{aligned}
$$

where $\left(G_{1}, \ldots, G_{n}\right)$ are $n$ i.i.d. randomly generated $N(0,1)$ random variables, $S(\boldsymbol{\beta}, \gamma, t, x)=n^{-1} \sum_{i=1}^{n} Y_{i}(t) e^{\boldsymbol{\beta}^{T} \mathbf{Z}_{i}^{*}(t)+\gamma^{T} \mathbf{Z}_{i}}$

$$
\begin{aligned}
& I\left(Z_{j i} \leq x\right), \mathbf{R}^{(k)}(\gamma, t)=n^{-1} \sum_{j=1}^{n} Y_{j}(t) \mathbf{Z}_{j}^{\otimes k} e^{\gamma^{T} \mathbf{Z}_{j}, \text { and }} \\
& \mathbf{B}(\boldsymbol{\beta}, \gamma, x)= n^{-1} \sum_{i=1}^{n} \int_{0}^{\tau} Y_{i}(u) \\
& \times \exp \left\{\boldsymbol{\beta}^{T} \mathbf{Z}_{i}^{*}(u)+\gamma^{T} \mathbf{Z}_{i}\right\} I\left(Z_{j i} \leq x\right) \\
& \times\left\{\mathbf{Z}_{i}^{*}(u)-\frac{\mathbf{S}^{(1)}(\boldsymbol{\beta}, \gamma, u)}{\mathbf{S}^{(0)}(\boldsymbol{\beta}, \gamma, u)}\right\} d \tilde{H}_{0}^{*}(u ; \boldsymbol{\beta}, \gamma) .
\end{aligned}
$$

One can plot $W_{j}(\cdot ; \tilde{\gamma})$, along with several realizations from $\tilde{W}_{j}(\cdot ; \tilde{\gamma})$, to graphically assess the functional form of the covariate. A more objective test can be developed based on $\tilde{s}_{j} \equiv \sup _{x}\left|W_{j}(\cdot ; \tilde{\gamma})\right|$

Note that we are fitting two models, one for the time to tumor, and the other for the monitoring time. Our primary interest is in model adequacy for (2.1) in the dependent monitoring scenario. Based on the estimation procedure described by equations (2.6) and (2.7), it is obvious that misspecification of the model in (2.4) will yield biased estimators for the regression coefficients in (2.1). In this case, assessing model adequacy for (2.1) has no meaning. Thus, before assessing model adequacy for (2.1), we need to check that our estimation procedure is providing consistent estimates of $\beta_{0}$. To do this requires checking model (2.4). Note that the reasons for model checking in the two instances, however, are different.

Suppose we are interested in assessing the functional form of a covariate in (2.1) for the dependent monitoring times situation. We propose the following sequential strategy for model checking:

1. Examine the overall fit of monitoring times model (2.4) using standard methods (e.g., Schoenfeld, 1982; Therneau, Grambsch, and Fleming, 1990; Lin et al., 1993). If the model form is deemed to be adequate, proceed to step 2; otherwise, refit a new model to the data.

2. Check the functional form of the covariate in the dependent monitoring model using the methods proposed here.

Note that we are performing a global test of model adequacy in the first step and a test for adequacy of a covariate at the second. While we have assumed in this article that the covariate vectors in the two models (2.1) and (2.4) are the same, this approach also works with different covariates in the two models. This will be demonstrated in Section 5 .

\section{Simulation Studies}

To assess the small-sample properties of the proposed methods, extensive simulation studies were conducted. In the ones reported here, we consider only a single covariate $Z$. We examined sample sizes $n=50,100$, and 200. Model (2.4) was used to generate monitoring times with $d \Lambda_{0}^{C}(t) \equiv \lambda_{0}^{C}(t)=0.5,1.0$, and 1.5 , and $\gamma_{0}=0.5$. This corresponds to dependent monitoring. For each simulation setting, 1000 samples were generated, and 1000 resamplings were used to calculate $p$-values for each sample.

For the simulation studies reported here, $n / 5$ subjects were assigned to one of five dose groups. The covariate $Z$ takes values $0,1,2,3$, and 4 . Failure times were generated from (2.1) with $\lambda_{0}(t) \equiv 1.0$ and $\beta=0.05$. The size of $\tilde{s}_{1}$ was assessed 
Table 1

Empirical powers of $\tilde{s}_{1}$

\begin{tabular}{|c|c|c|c|c|c|c|c|}
\hline \multirow[b]{2}{*}{$n$} & \multirow[b]{2}{*}{$\alpha$} & \multicolumn{3}{|c|}{ Correct model } & \multicolumn{3}{|c|}{ Incorrect model } \\
\hline & & $\lambda_{0}^{C}(t)=0.5$ & $\lambda_{0}^{C}(t)=1.0$ & $\lambda_{0}^{C}(t)=1.5$ & $\lambda_{0}^{C}(t)=0.5$ & $\lambda_{0}^{C}(t)=1.0$ & $\lambda_{0}^{C}(t)=1.5$ \\
\hline \multirow[t]{4}{*}{50} & 0.05 & 0.06 & 0.04 & 0.04 & 0.62 & 0.61 & 0.57 \\
\hline & 0.10 & 0.11 & 0.09 & 0.08 & 0.64 & 0.62 & 0.59 \\
\hline & 0.15 & 0.16 & 0.14 & 0.12 & 0.66 & 0.63 & 0.61 \\
\hline & 0.20 & 0.25 & 0.22 & 0.19 & 0.68 & 0.63 & 0.62 \\
\hline \multirow[t]{4}{*}{100} & 0.05 & 0.05 & 0.04 & 0.03 & 0.65 & 0.63 & 0.60 \\
\hline & 0.10 & 0.10 & 0.09 & 0.09 & 0.69 & 0.65 & 0.62 \\
\hline & 0.15 & 0.15 & 0.14 & 0.15 & 0.72 & 0.68 & 0.64 \\
\hline & 0.20 & 0.19 & 0.20 & 0.19 & 0.75 & 0.73 & 0.67 \\
\hline \multirow[t]{4}{*}{200} & 0.05 & 0.05 & 0.05 & 0.04 & 0.69 & 0.66 & 0.62 \\
\hline & 0.10 & 0.10 & 0.10 & 0.09 & 0.72 & 0.69 & 0.65 \\
\hline & 0.15 & 0.15 & 0.15 & 0.14 & 0.75 & 0.72 & 0.68 \\
\hline & 0.20 & 0.20 & 0.20 & 0.19 & 0.78 & 0.75 & 0.73 \\
\hline
\end{tabular}

Note: $\alpha$ denotes level of significance. "Correct model" refers to using $Z$ in the estimation procedure; "incorrect model" refers to using $Z^{*}$ in the estimation procedure. Failure times were generated from $(2.1)$ with $\lambda_{0}(t) \equiv 1.0$ and $\beta=0.05$. Monitoring times were generated from (2.4) with $\gamma_{0}=0.5$ and baseline hazard $\lambda_{0}^{C}(t)$.

by using $Z$ in the estimation procedures. The powers of the proposed method was assessed in two ways. For the first, the covariate $Z^{*}$ was used in estimation, where $Z^{*}=1$ if $Z>2$ and 0 otherwise. We also considered the two-stage procedure described at the end of Section 3. In the other scenario, $Z^{*}$ was used to generate data from (2.4) and $Z$ was used to generate data from (2.1). In the estimation procedure, $Z^{*}$ was used for both instances. We again compared the performance of $\tilde{s}_{1}$ and the proposed sequential methods. The results of these numerical studies are summarized in Table 1.

Based on these results, we find that the proposed methods perform reasonably well, at least for moderately larger

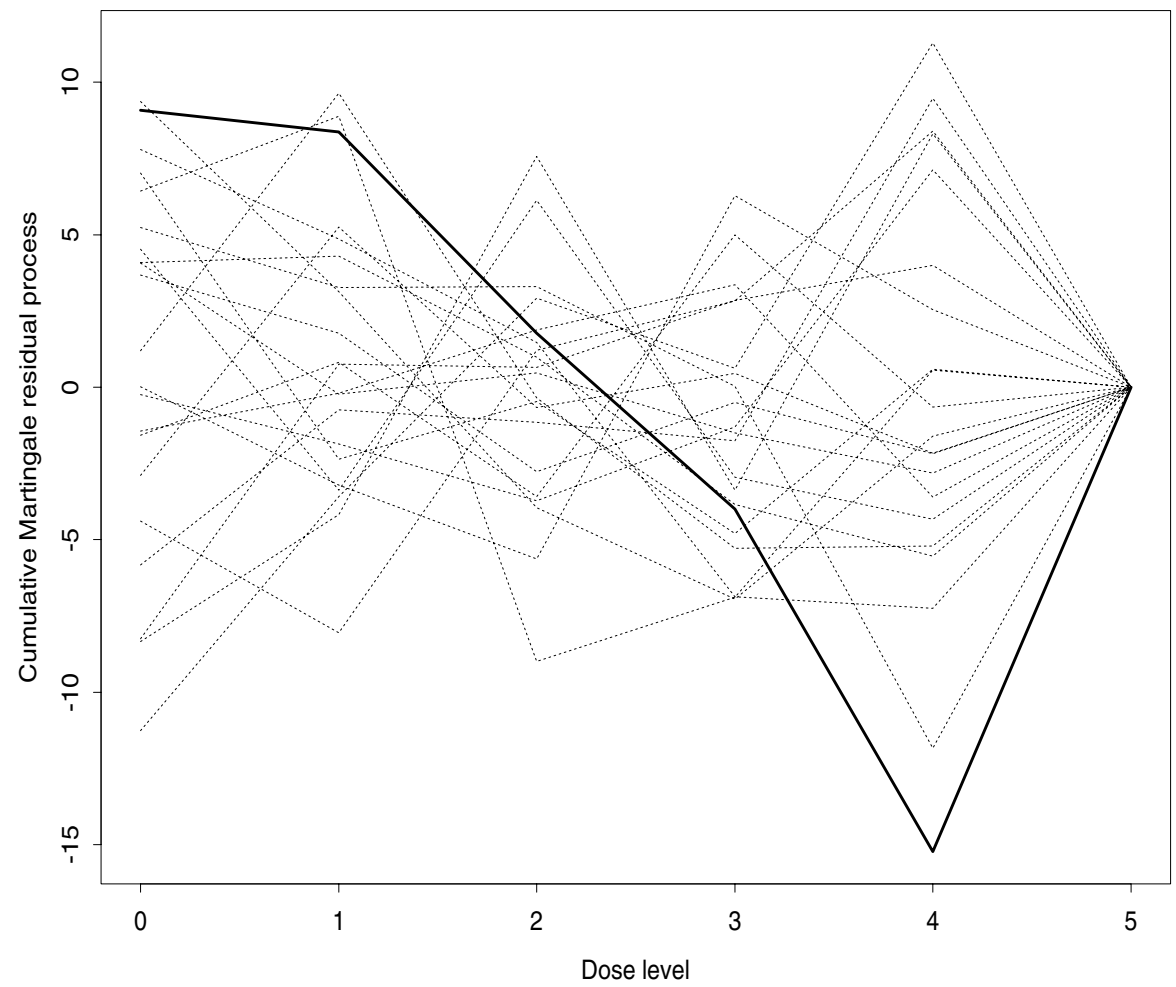

Figure 1. Cumulative sum residual process for assessing covariate form of dose for bile duct hyperplasia data with $Z^{*}$ in (2.4) and $Z$ in (2.1); solid line = observed process; dotted lines $=$ simulated processes. 


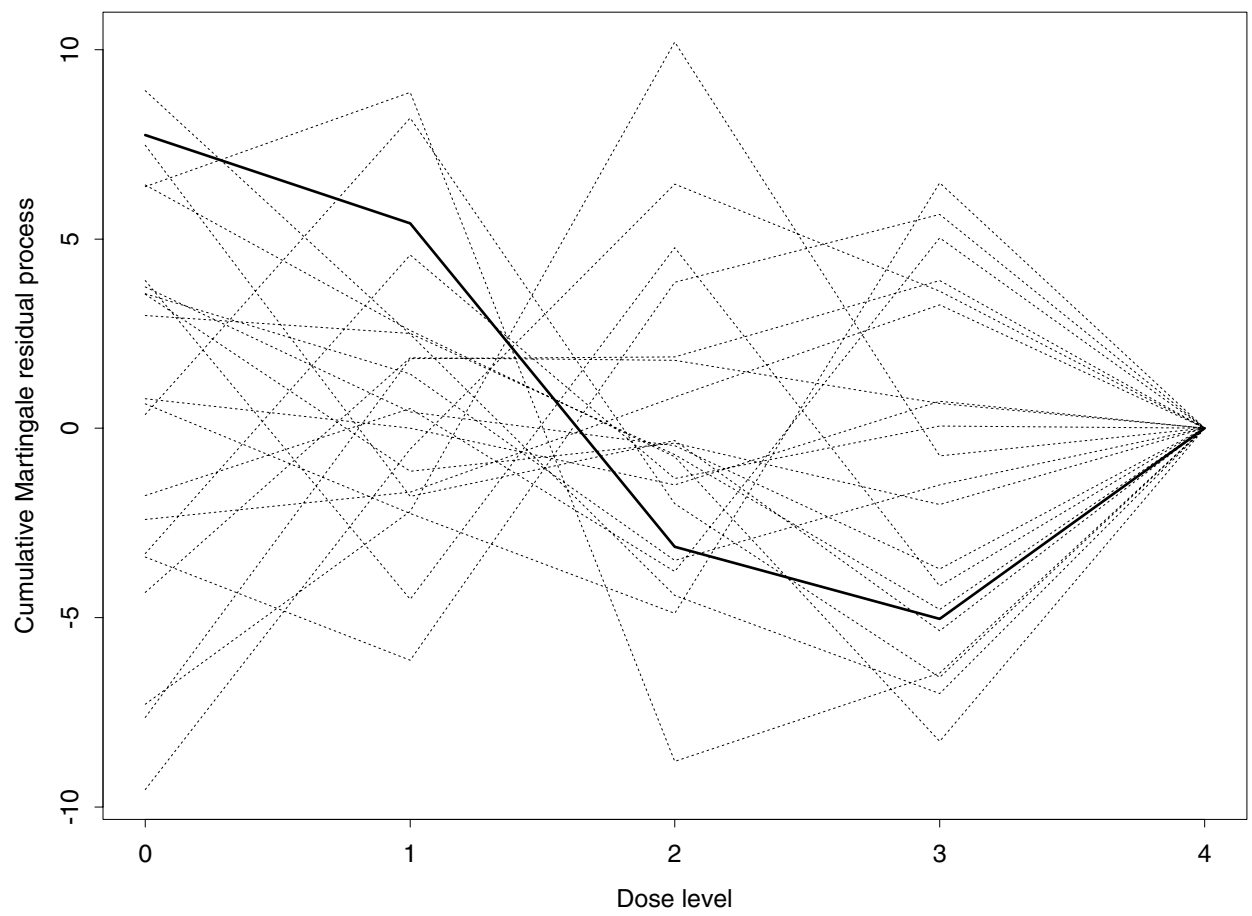

Figure 2. Cumulative sum residual process for assessing covariate form of dose for bile duct hyperplasia data with $Z^{*}$ in (2.4) and $Z^{*}$ in (2.1); solid line = observed process; dotted lines $=$ simulated processes.

sample sizes. For smaller sample sizes, the statistics tend to be somewhat unstable in terms of achieving the proper level of significance. This behavior diminishes in larger sample sizes. The method has good power.

\section{Bile Duct Hyperplasia Data}

We now revisit the bile duct data example from Section 1. In their analysis of these data, Dinse and Lagakos (1983) focus on the dose effect on tumor prevalence. Here, our interest is in the effect of treatment on time to tumor development, adjusting for other covariates. We consider model (2.1) for time to tumor with treatment, and sex $(0=$ female, $1=$ male $)$ as the covariates.

We first consider the model with a linear dose term and sex for both the monitoring times model (2.4) and the additivehazards model (2.1) for time to tumor. Using the sequential strategy proposed in Section 3, we first assess model adequacy for the monitoring times model. Based on the global procedure of Lin et al. (1993), the PH model for monitoring times is not appropriate ( $p$-value for the $\tilde{\mathrm{S}}$-type statistic based on 10,000 resamplings: 0.004$)$. We refit the data by transforming dose into covariate $Z^{*}$ for $(2.4): Z^{*}$ is the original dose if the actual dose level was at most 3.0, and 4 otherwise. We leave the dose as a linear term in model (2.1). Based on the model for monitoring times, the overall model is reasonably adequate ( $p$-value from the Lin et al. (1993), procedure $=$ $0.20)$. A plot of the martingale cumulative sum process for the time to tumor as a function of dose is given in Figure 1. The $p$-value for the $\tilde{\mathrm{S}}$-type statistic proposed in Section 3, based on 10,000 resamplings, is 0.005 . This suggests that the linear term for dose in the model (2.1) is not adequate. Suppose we now include $Z^{*}$ in both (2.1) and (2.4). The plot of the martingale cumulative sum process for the time to tumor is given in Figure 2. The $p$-value for the $\tilde{\mathrm{S}}$-type statistic proposed in Section 3, based on 10,000 resamplings, is 0.25 . This suggests that the transformation for dose in the model (2.1) is appropriate.

\section{Discussion}

While we have considered goodness of fit methods for checking covariate form, an extension of the ideas presented in the article can be used to check for the additive-hazards assumption with respect to each covariate component, as well as globally for model (2.1).

As was mentioned by Lin et al. (1998), the resulting estimators from their procedure are not semiparametrically efficient. In theory, it is possible to adapt the goodness-of-fit methods proposed here to efficient estimation procedures. For efficient estimation, see Ghosh (2001) and Martinussen and Scheike (2002). However, computation becomes more burdensome, because smoothing is required.

\section{ACKNOWLEDGEMENTS}

The author would like to thank Dr Gregg Dinse for the bile duct hyperplasia data and Dr Susan Murray for helpful comments on an earlier version of the manuscript. In addition, he would like to thank the associate editor and referee, whose comments improved the article.

\section{RÉSUMÉ}

Dans les expériences de tumorigénicité, un des problèmes posés est la mesure du délai jusqu'à apparition d'un événement,, qui n'est pas précisément observé et soumis à une 
censure par intervalle. Un des objectifs de ces type d'études est de modéliser de façon adéquate l'effet-dose sur un risque. Il est donc important de disposer de procédures permettant de tester l'adéquation du modèle. Alors que plusieurs procédures d'estimation ont été développées pour analyser ce type de données, peu de travaux ont été réalisés pour tester la validité de ces modèles. Dans cet article, nous proposons des méthodes numériques et graphiques pour les modèles à risques additifs afin d'analyser ces données d'état mesuré dans le temps, en prenant en compte le problème de la dépendance des temps de suivi du monitoring. Les propriétés de la méthode proposée sont étudiées par des études par simulation numérique, puis illustrées sur des données provenant d'une expérience de tumorigénicité.

\section{REFERENCES}

Breslow, N. and Day, N. E. (1980). Statistical Methods in Cancer Research, Volume 1: The Analysis of Case-Control Studies. Lyon: World Health Organization.

Breslow, N. and Day, N. E. (1987). Statistical Methods in Cancer Research, Volume 2: The Design and Analysis of Cohort Studies. Lyon: World Health Organization.

Cox, D. R. (1972). Regression models and life tables (with discussion). Journal of the Royal Statistical Society, Series B 34, 187-220.

Dinse, G. E. and Lagakos, S. W. (1983). Regression analysis of tumour prevalence data. Applied Statistics 32, 236248.

Farrington, C. P. (2000). Residuals for proportional hazards models with interval-censored survival data. Biometrics 56, 473-482.

Ghosh, D. (2001). Efficiency considerations in the additive hazards model with current status data. Statistica Neerlandica 55, 367-376.

Hoel, D. G. and Walburg, H. E. (1972). Statistical analysis of survival experiments. Journal of the National Cancer Institute 45, 361-372.

Lin, D. Y. and Ying, Z. (1994). Semiparametric analysis of the additive risk model. Biometrika 81, 61-71.

Lin, D. Y., Wei, L. J. and Ying, Z. (1993). Checking the Cox model with cumulative sums of martingale-based residuals. Biometrika 80, 557-572.

Lin, D. Y., Oakes, D., and Ying, Z. (1998). Additive hazards regression with current status data. Biometrika 85, 289298.

Lin, D. Y., Wei, L. J., Yang, I. and Ying, Z. (2000). Semiparametric regression for the mean and rate functions of recurrent events. Journal of the Royal Statistical Society, Series B 62, 711-730.

Martinussen, T. and Scheike, T. H. (2002). Efficient estimation in additive hazards regression with current status data. Biometrika 89, 649-658.

Schoenfeld, D. (1982). Partial residuals for the proportional hazards regression model. Biometrika 69, 239-241.

Therneau, T. M., Grambsch, P. M. and Fleming, T. R., (1990). Martingale-based residuals for survival models. Biometrika 77, 147-160.

Younes, N. and Lachin, J. (1997). Link-based models for survival data with interval and continuous time censoring. Biometrics 53, 1199-1211.

Received February 2002. Revised November 2002. Accepted January 2003. 